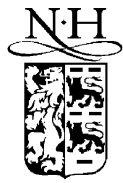

ELSEVIER

\title{
Exact phase shifts for atom interferometry
}

\author{
Ch. Antoine ${ }^{\mathrm{a}, *}$, Ch.J. Bordé $\mathrm{a,b,*}$ \\ a Equipe de Relativité Gravitation et Astrophysique, LERMA, CNRS-Observatoire de Paris, Université Pierre et Marie Curie, \\ 4 place Jussieu, 75005 Paris, France \\ b Laboratoire de Physique des Lasers, UMR 7538 CNRS, Université Paris Nord, 99 avenue J.-B. Clément, 93430 Villetaneuse, France
}

Received 28 October 2002; accepted 8 November 2002

Communicated by P.R. Holland

\begin{abstract}
In the case of an external Hamiltonian at most quadratic in position and momentum operators, we use the $\mathrm{ABCD} \xi$ formulation of atom optics to establish an exact analytical phase shift expression for atom interferometers with arbitrary spatial or temporal beam splitter configurations. This result is expressed in terms of coordinates and momenta of the wave packet centers at the interaction vertices only.
\end{abstract}

() 2002 Elsevier Science B.V. All rights reserved.

\section{Introduction}

Recently atom interferometers [1] have been described by the $\mathrm{ABCD} \xi$ formalism of Gaussian atom optics [2,3] which yields an exact formulation of phase shifts taking into account the wave packet structure of atom waves.

For the theory of atom interferometers two basic stages are required:

1. A proper description of the propagation of wave packets between the beam splitters;

2. An adequate modelization of the beam splitters themselves.

The first stage is achieved through the $\mathrm{ABCD} \xi$ theorem whose main results are briefly recalled in Section 2. The second problem is addressed by the ttt theorem which provides a simple model for the phase introduced by the splitting process.

In this Letter we give a compact way to express the atom interferometer phase shifts in terms of the coordinates and momenta of the wave packet centers only. For this purpose we derive two new theorems (the four endpoints theorem and the phase shift formula) valid for a Hamiltonian at most quadratic in position and momentum operators.

\footnotetext{
* Corresponding authors.

E-mail addresses: antoinec@ccr.jussieu.fr (Ch. Antoine), chbo@ccr.jussieu.fr (Ch.J. Bordé).
} 


\section{The $\mathrm{ABCD} \xi$ theorem}

In this framework we consider a Hamiltonian which is the sum of an internal Hamiltonian $H_{0}$ (with eigenvalues written with rest masses $m_{i}$ ) and of an external Hamiltonian $H_{\text {ext }}$ :

$$
H_{\mathrm{ext}}=\frac{1}{2 m} \overrightarrow{p_{\mathrm{op}}} \cdot \vec{g}(t) \cdot \overrightarrow{p_{\mathrm{op}}}-\frac{m}{2} \overrightarrow{q_{\mathrm{op}}} \cdot \vec{\gamma}(t) \cdot \overrightarrow{q_{\mathrm{op}}}-\vec{\Omega}(t) \cdot\left(\overrightarrow{q_{\mathrm{op}}} \times \overrightarrow{p_{\mathrm{op}}}\right)-m \vec{g}(t) \cdot \overrightarrow{q_{\mathrm{op}}},
$$

where one recognizes several usual gravito-inertial effects: rotation in $\vec{\Omega}(t)$, gravity in $\vec{g}(t)$, gradient of gravity in $\vec{\gamma}(t), \ldots$ and where $\vec{g}(t)$ is usually taken equal to the unity tensor in the absence of gravitational wave.

For a wave packet $\psi\left(q, t_{1}\right)=w p\left(t_{1}, q-q_{1}, p_{1}, X_{1}, Y_{1}\right)$, where $q_{1}$ is the initial mean position of the wave packet, $p_{1}$ its initial mean momentum, and $\left(X_{1}, Y_{1}\right)$ its initial complex width parameters in phase space, one obtains the $\mathrm{ABCD} \xi$ theorem [2]:

$$
\begin{aligned}
\psi\left(q, t_{2}\right) & =\int d^{3} q^{\prime} \cdot K\left(q, t_{2}, q^{\prime}, t_{1}\right) \cdot w p\left(t_{1}, q^{\prime}-q_{1}, p_{1}, X_{1}, Y_{1}\right) \\
& =e^{\frac{i}{\hbar} S_{\mathrm{cl}}\left(t_{2}, t_{1}, q_{1}, p_{1}\right)} \cdot w p\left(t_{2}, q-q_{2}, p_{2}, X_{2}, Y_{2}\right),
\end{aligned}
$$

where $K$ and $S_{\mathrm{cl}}$ are, respectively, the quantum propagator and the classical action, and where $q_{2}, p_{2}, X_{2}, Y_{2}$ obey the ABCD law ( $G$ and $\mathcal{R}$ are the representative matrices of $\vec{g}(t)$ and of the rotation operator corresponding to $\vec{\Omega}(t)$, and we write $A_{21}$ instead of $A\left(t_{2}, t_{1}\right)$ for simplicity):

$$
\begin{aligned}
& \left(\begin{array}{c}
q_{2} \\
p_{2} / m
\end{array}\right)=\left(\begin{array}{c}
\mathcal{R}_{21} \cdot \xi_{21} \\
G_{2}^{-1} \cdot \mathcal{R}_{21} \cdot \dot{\xi}_{21}
\end{array}\right)+\left(\begin{array}{ll}
A_{21} & B_{21} \\
C_{21} & D_{21}
\end{array}\right) \cdot\left(\begin{array}{c}
q_{1} \\
p_{1} / m
\end{array}\right), \\
& \left(\begin{array}{c}
X_{2} \\
Y_{2}
\end{array}\right)=\left(\begin{array}{ll}
A_{21} & B_{21} \\
C_{21} & D_{21}
\end{array}\right) \cdot\left(\begin{array}{c}
X_{1} \\
Y_{1}
\end{array}\right) .
\end{aligned}
$$

For example, the phase of a Gaussian wave packet is (for simplicity we shall omit the transposition sign $\sim$ on matrix representations of vectors):

$$
S_{\mathrm{cl}}\left(t_{2}, t_{1}, q_{1}, p_{1}\right) / \hbar+p_{2} \cdot\left(q-q_{2}\right) / \hbar+\frac{m}{2 \hbar}\left(q-q_{2}\right) \cdot \operatorname{Re}\left(Y_{2} \cdot X_{2}^{-1}\right) \cdot\left(q-q_{2}\right)
$$

and in this case the main phase shift between $t_{1}$ and $t_{2}$ is equal to:

$$
S_{\mathrm{cl}}\left(t_{2}, t_{1}, q_{1}, p_{1}\right) / \hbar+p_{1} . q_{1} / \hbar-p_{2} . q_{2} / \hbar \text {. }
$$

\section{The ttt theorem}

When the dispersive nature of a beam splitter is neglected (i.e., the wave packet structure is preserved), its effect may be summarized by the introduction of both a phase and an amplitude factor (see [13] and [4] for a detailed proof):

$$
\left.M_{b a} \cdot e^{-i\left(\omega^{*} t^{*}-k^{*}\right.} \cdot q^{*}+\varphi^{*}\right),
$$

where $t^{*}$ and $q^{*}$ depend on $t_{A}$ and $q_{A}$, the mean time and position of the electromagnetic wave used as a beam splitter. 
For a temporal beam splitter:

$$
\begin{aligned}
t^{*} & \equiv t_{A}, \\
q^{*} & \equiv q_{\mathrm{cl}}\left(t_{A}\right), \\
k^{*} & \equiv k, \\
\omega^{*} & =\omega, \\
\varphi^{*} & \equiv \varphi \text { (laser phase) }
\end{aligned}
$$

For a spatial beam splitter:

$$
\begin{aligned}
q^{*} & \equiv q_{A}, \\
t^{*} \text { such that } q_{\mathrm{cl}}\left(t^{*}\right) & \equiv q_{A}, \\
k^{*} & \equiv k+\delta k, \\
\omega^{*} & =\omega, \\
\varphi^{*} & \equiv \varphi+\delta \varphi,
\end{aligned}
$$

where $q_{\mathrm{cl}}$ is the central position of the input atomic wave packet (equal to the classical position because of Ehrenfest theorem), where $\delta k$ is the additional momentum transferred to the excited atoms out of resonance, and where $\delta \varphi$ is a laser phase: $\delta \varphi \equiv-\delta k \cdot q_{A}$ (see [4]).

Let us emphasize that these calculations do not rely on the assumption that the splitter is infinitely thin or that the atom trajectories are classical.

\section{The four end-points theorem for a Hamiltonian at most quadratic in position and momentum operators}

We shall cut any interferometer into as many slices as there are interactions on either arm and thus obtain several path pieces (see Section 5). From now on we shall consider systematically pairs of these homologous paths (see Fig. 1) in the case of a Hamiltonian at most quadratic.

These two classical trajectories are labelled by their corresponding mass $\left(m_{\alpha}\right.$ and $\left.m_{\beta}\right)$, their initial position and momentum $\left(q_{\alpha 1}, p_{\alpha 1}, q_{\beta 1}\right.$ and $\left.p_{\beta 1}\right)$ and their common drift time $T=t_{2}-t_{1}$.

Before establishing the first new theorem let us consider the expression of the classical action for the $\alpha$ path (see [2]):

$$
\begin{aligned}
& \frac{S_{\mathrm{cl}}\left(t_{2}, t_{1}, q_{\alpha 1}, p_{\alpha 1}\right)}{m_{\alpha}} \\
& \quad=\dot{\xi} \cdot \widetilde{\mathcal{R}} \cdot G^{-1} \cdot\left(A \cdot q_{\alpha 1}+B \cdot p_{\alpha 1} / m_{\alpha}\right)+\int_{t_{1}}^{t_{2}} \frac{L}{m_{\alpha}} d t+q_{\alpha 1} \cdot \frac{\widetilde{A} C}{2} \cdot q_{\alpha 1}+\frac{p_{\alpha 1}}{m_{\alpha}} \cdot \frac{\widetilde{B} D}{2} \cdot \frac{p_{\alpha 1}}{m_{\alpha}}+\frac{p_{\alpha 1}}{m_{\alpha}} \cdot \widetilde{B} C \cdot q_{\alpha 1},
\end{aligned}
$$

where $\vec{\xi}$ and $L$ depend on $\vec{g}(t)$ (see [2] for notations).

This expression can be rewritten as:

$$
\begin{aligned}
& \frac{S_{\mathrm{cl}}\left(t_{2}, t_{1}, q_{\alpha 1}, p_{\alpha 1}\right)}{m_{\alpha}} \\
& \quad=\frac{p_{\alpha 2}}{2 m_{\alpha}} \cdot q_{\alpha 2}-\frac{p_{\alpha 1}}{2 m_{\alpha}} \cdot q_{\alpha 1}-\frac{1}{2} \dot{\xi} \cdot \widetilde{\mathcal{R}} \cdot G^{-1} \cdot \mathcal{R} \cdot \xi+\int_{t_{1}}^{t_{2}} \frac{L}{m_{\alpha}} d t+\frac{1}{2} \dot{\xi} \cdot \widetilde{\mathcal{R}} \cdot G^{-1} \cdot q_{\alpha 2}-\frac{1}{2} \xi \cdot \widetilde{\mathcal{R}} \cdot \frac{p_{\alpha 2}}{m_{\alpha}}
\end{aligned}
$$




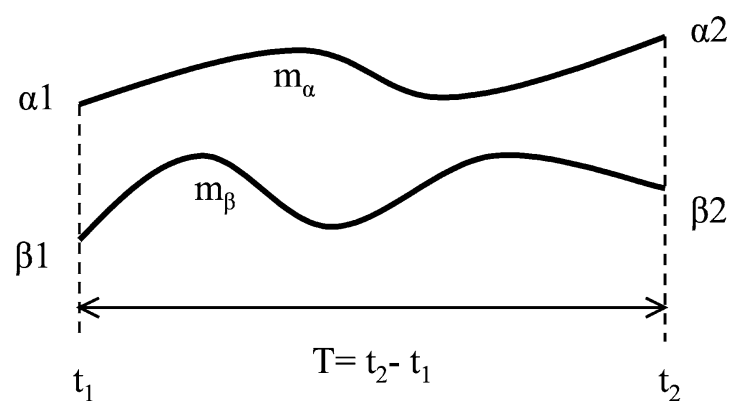

Fig. 1. A pair of homologous paths.

with the help of the definition of $q_{\alpha 2}$ and $\frac{p_{\alpha 2}}{m_{\alpha}}$ (see formula (3)). Then we can use the $\beta$ path to replace $\dot{\xi} . \widetilde{\mathcal{R}} \cdot G^{-1}$ with:

$$
\dot{\xi} . \widetilde{\mathcal{R}} \cdot G^{-1}=\frac{p_{\beta 2}}{m_{\beta}}-C \cdot q_{\beta 1}-D \cdot \frac{p_{\beta 1}}{m_{\beta}} .
$$

Consequently we get:

$$
\frac{S_{\mathrm{cl}}\left(t_{2}, t_{1}, q_{\alpha 1}, p_{\alpha 1}\right)}{m_{\alpha}}=\frac{1}{2}\left(\frac{p_{\alpha 2}}{m_{\alpha}}+\frac{p_{\beta 2}}{m_{\beta}}\right) \cdot q_{\alpha 2}-\frac{1}{2}\left(\frac{p_{\alpha 1}}{m_{\alpha}}+\frac{p_{\beta 1}}{m_{\beta}}\right) \cdot q_{\alpha 1}+h\left(t_{2}, t_{1}\right)+f(\alpha, \beta),
$$

where $h\left(t_{2}, t_{1}\right)$ is independent of positions and momenta and where $f(\alpha, \beta)=f(\beta, \alpha)$. The same conclusion holds for the expression of $S_{\mathrm{cl}}\left(t_{2}, t_{1}, q_{\beta 1}, p_{\beta 1}\right) / m_{\beta}$ which is obtained by exchanging $\alpha$ and $\beta$. Finally, we arrive at the first new theorem (a more general demonstration starting with Hamilton principal functions is given in Appendix A):

\section{Theorem 1.}

$$
\begin{aligned}
& \frac{S_{\mathrm{cl}}\left(t_{2}, t_{1}, q_{\alpha 1}, p_{\alpha 1}\right)}{m_{\alpha}}-\frac{1}{2}\left(\frac{p_{\alpha 2}}{m_{\alpha}}+\frac{p_{\beta 2}}{m_{\beta}}\right) \cdot q_{\alpha 2}+\frac{1}{2}\left(\frac{p_{\alpha 1}}{m_{\alpha}}+\frac{p_{\beta 1}}{m_{\beta}}\right) \cdot q_{\alpha 1} \\
& =\frac{S_{\mathrm{cl}}\left(t_{2}, t_{1}, q_{\beta 1}, p_{\beta 1}\right)}{m_{\beta}}-\frac{1}{2}\left(\frac{p_{\alpha 2}}{m_{\alpha}}+\frac{p_{\beta 2}}{m_{\beta}}\right) \cdot q_{\beta 2}+\frac{1}{2}\left(\frac{p_{\alpha 1}}{m_{\alpha}}+\frac{p_{\beta 1}}{m_{\beta}}\right) \cdot q_{\beta 1}
\end{aligned}
$$

or equivalently:

$$
\begin{aligned}
& \frac{S_{\mathrm{cl}}\left(t_{2}, t_{1}, q_{\alpha 1}, p_{\alpha 1}\right)}{m_{\alpha}}-\frac{p_{\alpha 2}}{m_{\alpha}} \cdot q_{\alpha 2}+\frac{p_{\alpha 1}}{m_{\alpha}} \cdot q_{\alpha 1}-\left[\frac{S_{\mathrm{cl}}\left(t_{2}, t_{1}, q_{\beta 1}, p_{\beta 1}\right)}{m_{\beta}}-\frac{p_{\beta 2}}{m_{\beta}} \cdot q_{\beta 2}+\frac{p_{\beta 1}}{m_{\beta}} \cdot q_{\beta 1}\right] \\
& \quad=\left(\frac{p_{\beta 2}}{m_{\beta}}-\frac{p_{\alpha 2}}{m_{\alpha}}\right) \cdot\left(\frac{q_{\alpha 2}+q_{\beta 2}}{2}\right)-\left(\frac{p_{\beta 1}}{m_{\beta}}-\frac{p_{\alpha 1}}{m_{\alpha}}\right) \cdot\left(\frac{q_{\alpha 1}+q_{\beta 1}}{2}\right)
\end{aligned}
$$

which will give the main part of the phase shift expressed with the half sums of the coordinates and the momenta of the four end-points only.

In the case of identical masses $\left(m_{\alpha}=m_{\beta}\right)$ this expression simplifies to:

$$
\begin{aligned}
& S_{\mathrm{cl}}\left(t_{2}, t_{1}, q_{\alpha 1}, p_{\alpha 1}\right)-p_{\alpha 2} \cdot q_{\alpha 2}+p_{\alpha 1} \cdot q_{\alpha 1}-\left[S_{\mathrm{cl}}\left(t_{2}, t_{1}, q_{\beta 1}, p_{\beta 1}\right)-p_{\beta 2} \cdot q_{\beta 2}+p_{\beta 1} \cdot q_{\beta 1}\right] \\
& \quad=\left(p_{\beta 2}-p_{\alpha 2}\right) \cdot\left(\frac{q_{\alpha 2}+q_{\beta 2}}{2}\right)-\left(p_{\beta 1}-p_{\alpha 1}\right) \cdot\left(\frac{q_{\alpha 1}+q_{\beta 1}}{2}\right) .
\end{aligned}
$$




\section{The phase shift formula for a Hamiltonian at most quadratic in position and momentum operators}

In this section we draw on the results of previous sections to establish the interferometer phase shift expression for an arbitrary beam splitters configuration.

For a sequence of pairs of homologous paths (an interferometer geometry) (see Fig. 2) one can infer the general sum for the main coordinate-dependent part of the global phase shift:

$$
\frac{p_{\beta D}-p_{\alpha D}}{\hbar} \cdot\left(q-\frac{q_{\alpha D}+q_{\beta D}}{2}\right)-\frac{p_{\alpha 1}+p_{\beta 1}}{2 \hbar} \cdot\left(q_{\beta 1}-q_{\alpha 1}\right)+\sum_{i=1}^{N}\left(k_{\beta i}-k_{\alpha i}\right) \cdot \frac{q_{\alpha i}+q_{\beta i}}{2} .
$$

If now we take into account the other terms of the phase shift, we finally obtain the following result (given here for a Gaussian wave packet):

\section{Theorem 2.}

$$
\begin{aligned}
\Delta \phi\left(q, t_{N+1} \equiv t_{D}\right) & \\
= & \left(p_{\beta D}-p_{\alpha D}\right) \cdot\left(q-\frac{q_{\alpha D}+q_{\beta D}}{2}\right) / \hbar-\frac{p_{\alpha 1}+p_{\beta 1}}{2 \hbar} \cdot\left(q_{\beta 1}-q_{\alpha 1}\right) \\
+ & \sum_{i=1}^{N}\left[\left(k_{\beta i}-k_{\alpha i}\right) \cdot \frac{q_{\alpha i}+q_{\beta i}}{2}-\left(\omega_{\beta i}-\omega_{\alpha i}\right) \cdot t_{i}+\varphi_{\beta i}-\varphi_{\alpha i}\right] \\
+ & \sum_{i=1}^{N}\left(\frac{m_{\beta i}-m_{\alpha i}}{2 \hbar}\right) \cdot\left\{\left(\frac{S_{\alpha i}}{m_{\alpha i}}+\frac{p_{\alpha, i+1}}{2 m_{\alpha i}} \cdot\left(q_{\beta, i+1}-q_{\alpha, i+1}\right)-\frac{p_{\alpha i}+\hbar k_{\alpha i}}{2 m_{\alpha i}} \cdot\left(q_{\beta i}-q_{\alpha i}\right)\right)\right. \\
& \left.\quad+\left(\frac{S_{\beta i}}{m_{\beta i}}+\frac{p_{\beta, i+1}}{2 m_{\beta i}} \cdot\left(q_{\alpha, i+1}-q_{\beta, i+1}\right)-\frac{p_{\beta i}+\hbar k_{\beta i}}{2 m_{\beta i}} \cdot\left(q_{\alpha i}-q_{\beta i}\right)\right)\right\} \\
+ & \frac{m_{\beta, N}}{2 \hbar}\left(q-q_{\beta D}\right) \cdot \operatorname{Re}\left(Y_{D} \cdot X_{D}^{-1}\right) \cdot\left(q-q_{\beta D}\right)-\frac{m_{\alpha, N}}{2 \hbar}\left(q-q_{\alpha D}\right) \cdot \operatorname{Re}\left(Y_{D} \cdot X_{D}^{-1}\right) \cdot\left(q-q_{\alpha D}\right),
\end{aligned}
$$

where $S_{\alpha i} \equiv S_{\mathrm{cl}}\left(t_{i+1}, t_{i}, q_{\alpha i}, p_{\alpha i}+\hbar k_{\alpha i}, m_{\alpha i}\right)$.

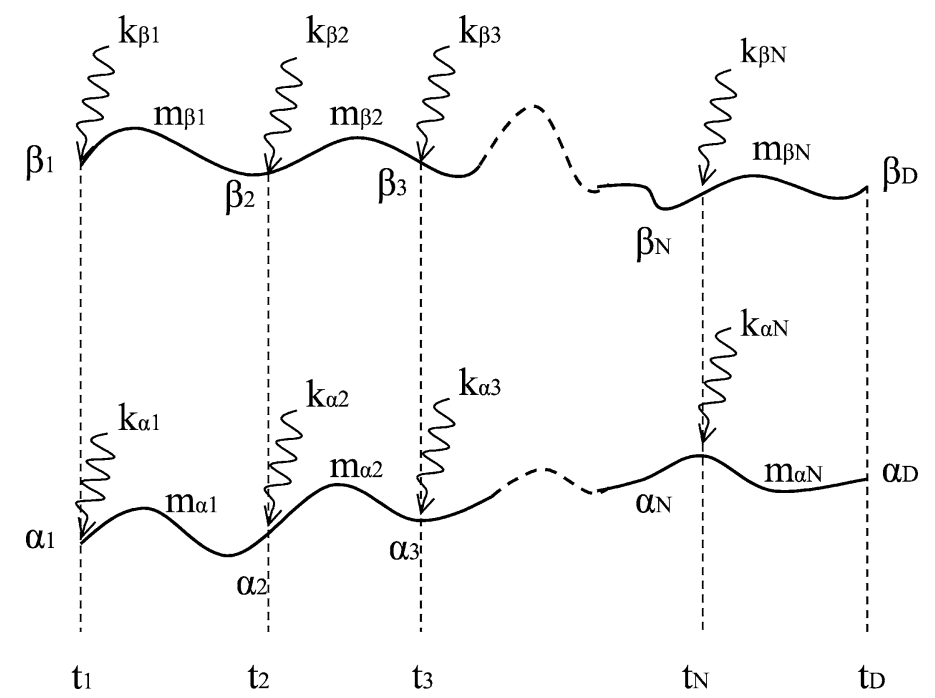

Fig. 2. Interferometer geometry sliced into pairs of homologous paths between interactions on either arm (when an interaction occurs only on one arm the corresponding $k$ on the other arm is set $=0$ ). 
This basic formula is valid for a time-dependent Hamiltonian and takes into account all the mass differences which may occur. It allows to calculate exactly the phase shift for all the interferometer geometries which can be sliced as above: symmetrical Ramsey-Bordé (Mach-Zehnder), atomic fountain clocks,.... All these particular cases will be detailed in forthcoming papers (see [6]).

Let us point out that the nature (temporal or spatial) of beam splitters leads to different slicing of the paths. In the spatial case, indeed, the number of different $t_{i}^{*}$ may be twice as great as in the temporal case (see the definition of $t_{i}^{*}$ in these two different cases in Section 3).

\section{Phase shift after spatial integration}

In any interferometer one has to integrate spatially the output wave packet over the detection region. With Gaussian wave packets this integration leads to a mid-point theorem [3,6]: "The first term of $\Delta \phi\left(q, t_{D}\right)$ disappears when the spatial integration is performed".

Furthermore the terms which depend on the wave packets structure $(Y$ and $X)$ vanish when $m_{\beta, N}=m_{\alpha, N}$ (which is always the case). One obtains finally:

$$
\begin{aligned}
\Delta \phi\left(t_{D}\right)= & -\frac{p_{\alpha 1}+p_{\beta 1}}{2 \hbar} \cdot\left(q_{\beta 1}-q_{\alpha 1}\right) \\
& +\sum_{i=1}^{N}\left[\left(k_{\beta i}-k_{\alpha i}\right) \cdot \frac{q_{\alpha i}+q_{\beta i}}{2}-\left(\omega_{\beta i}-\omega_{\alpha i}\right) \cdot t_{i}+\varphi_{\beta i}-\varphi_{\alpha i}\right] \\
+ & \sum_{i=1}^{N}\left(\frac{m_{\beta i}-m_{\alpha i}}{2 \hbar}\right) \cdot\left\{\left(\frac{S_{\alpha i}}{m_{\alpha i}}+\frac{p_{\alpha, i+1}}{2 m_{\alpha i}} \cdot\left(q_{\beta, i+1}-q_{\alpha, i+1}\right)-\frac{p_{\alpha i}+\hbar k_{\alpha i}}{2 m_{\alpha i}} \cdot\left(q_{\beta i}-q_{\alpha i}\right)\right)\right. \\
& \left.+\left(\frac{S_{\beta i}}{m_{\beta i}}+\frac{p_{\beta, i+1}}{2 m_{\beta i}} \cdot\left(q_{\alpha, i+1}-q_{\beta, i+1}\right)-\frac{p_{\beta i}+\hbar k_{\beta i}}{2 m_{\beta i}} \cdot\left(q_{\alpha i}-q_{\beta i}\right)\right)\right\} .
\end{aligned}
$$

\section{Identical masses and symmetrical case}

The case of identical masses is an important approximation which is commonly used for the modelization of many devices like gravimeters and gyrometers [7-9]. If $m_{\alpha i}=m_{\beta i}=m, \forall i$, this general phase shift becomes:

$$
\Delta \phi\left(t_{D}\right)=-\frac{p_{\alpha 1}+p_{\beta 1}}{2 \hbar} \cdot\left(q_{\beta 1}-q_{\alpha 1}\right)+\sum_{i=1}^{N}\left(k_{\beta i}-k_{\alpha i}\right) \cdot \frac{q_{\alpha i}+q_{\beta i}}{2}+\sum_{i=1}^{N}\left[\varphi_{\beta i}-\varphi_{\alpha i}-\left(\omega_{\beta i}-\omega_{\alpha i}\right) . t_{i}\right] .
$$

We can also specify the form of this phase shift when the interferometer geometry is symmetrical (see Fig. 3). This symmetry is expressed as: $k_{\beta i}+k_{\alpha i}=0, \forall i \in[2, N-1]$, i.e., it is a symmetry with respect to the direction of the particular vector: $p_{\text {initial }}+\hbar k_{\text {initial }} / 2$.

Consequently:

$$
\Delta \phi\left(t_{N}\right)=k_{1} \cdot q_{1}+2 \sum_{i=2}^{N-1} k_{i} \cdot \frac{q_{\alpha i}+q_{\beta i}}{2}+k_{N} \cdot \frac{q_{\alpha N}+q_{\beta N}}{2}-\sum_{i=1}^{N}\left(\varphi_{\beta i}-\varphi_{\alpha i}\right) .
$$




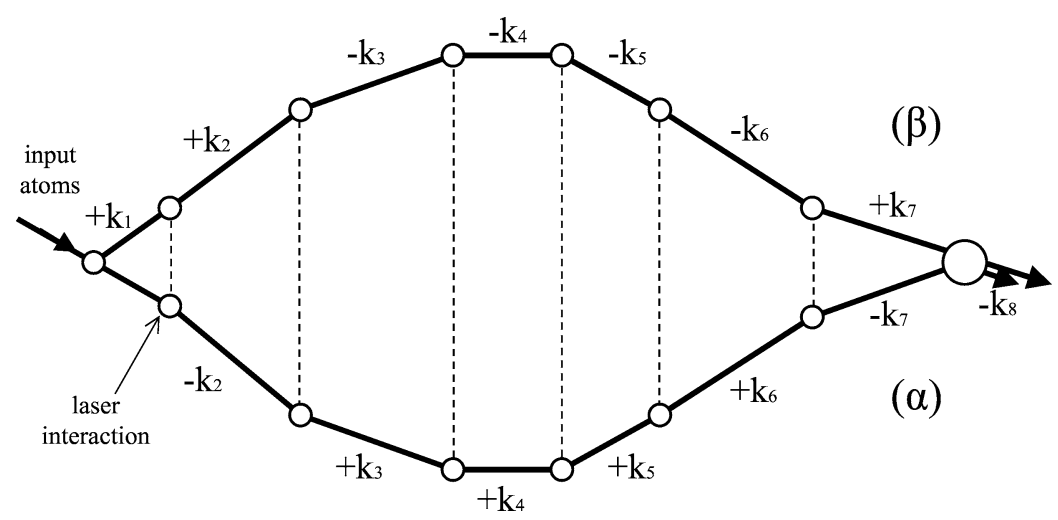

Fig. 3. A typical symmetrical interferometer.

But $\forall i \in[1, N-1]$ :

$$
\begin{aligned}
\frac{q_{\alpha, i+1}+q_{\beta, i+1}}{2} & =\xi_{i+1, i}+A_{i+1, i} \cdot \frac{q_{\alpha i}+q_{\beta i}}{2}+\frac{B_{i+1, i}}{m} \cdot \frac{p_{\alpha i}+p_{\beta i}}{2} \\
& =\xi_{i+1,1}+A_{i+1,1} \cdot q_{1}+\frac{B_{i+1,1}}{m} \cdot\left(p_{1}+\frac{\hbar k_{1}}{2}\right) \equiv Q\left(t_{i+1}\right)
\end{aligned}
$$

which can be calculated with the $\mathrm{ABCD} \xi$ law.

It depends only on $q_{1}$ ("initial position") and $p_{1}+\frac{\hbar k_{1}}{2}$ ("Bragg initial momentum").

Therefore:

$$
\Delta \phi\left(t_{N}\right)=\sum_{i=1}^{N}\left(k_{\beta i}-k_{\alpha i}\right) \cdot Q\left(t_{i}\right)-\sum_{i=1}^{N}\left(\varphi_{\beta i}-\varphi_{\alpha i}\right)
$$

which has a very simple form when the Bragg condition $p_{1}+\frac{\hbar k_{1}}{2}=0$ is satisfied.

\section{Conclusion}

In this Letter we have used the $\mathrm{ABCD} \xi$ formulation of atom optics and the ttt theorem to establish two theorems valid for a time-dependent Hamiltonian at most quadratic in position and momentum operators. The first one gives a compact expression of the action difference between two homologous paths. The second one gives an analytical expression of the global phase shift for atom interferometers in the case of such a Hamiltonian.

Consequently this analytical expression provides a simple way to calculate exactly the phase shift in this case, and then one can calculate perturbatively the effect of a higher-order term in the external Hamiltonian (necessary for space missions like HYPER [10]). For example, one can calculate exactly the global phase shift due to gravity plus a gradient of gravity plus a rotation, and then calculate perturbatively the effect of a gradient of gradient of gravity. These calculations and the application to specific cases (gravimeters, gyrometers, atomic clocks, ...) will be detailed in a forthcoming article [6] where we recover well-known perturbative results $([5,9,11,12])$ from exact expressions.

\section{Appendix A}

In the case of a Hamiltonian at most quadratic in position and momentum operators, the Hamilton principal functions concerning two pairs of homologous points are also at most quadratic in positions (owing to the 
Hamilton-Jacobi equation, see [2]):

$$
\begin{aligned}
& S_{\alpha}\left(q_{\alpha 1}, q_{\alpha 2}\right) / m_{\alpha}=a+b \cdot q_{\alpha 1}+c . q_{\alpha 2}+q_{\alpha 1} \cdot d \cdot q_{\alpha 1}+q_{\alpha 1} . e \cdot q_{\alpha 2}+q_{\alpha 2} \cdot f \cdot q_{\alpha 2}, \\
& S_{\beta}\left(q_{\beta 1}, q_{\beta 2}\right) / m_{\beta}=a+b \cdot q_{\beta 1}+c . q_{\beta 2}+q_{\beta 1} . d \cdot q_{\beta 1}+q_{\beta 1} . e \cdot q_{\beta 2}+q_{\beta 2} \cdot f \cdot q_{\beta 2},
\end{aligned}
$$

where $a$ is a scalar, $b$ and $c$ are vectors, and $d, e$ and $f$ are matrices (see [2]).

We can define $p_{\alpha 1}, p_{\alpha 2}, p_{\beta 1}, p_{\beta 2}$ such that

$$
\begin{aligned}
\frac{p_{\alpha 1}}{m_{\alpha}} & \equiv-\nabla_{q_{\alpha 1}}\left(\frac{S_{\alpha}}{m_{\alpha}}\right)=-b-2 d . q_{\alpha 1}-e . q_{\alpha 2}, \\
\frac{p_{\alpha 2}}{m_{\alpha}} & \equiv \nabla_{q_{\alpha 2}}\left(\frac{S_{\alpha}}{m_{\alpha}}\right)=c+2 f . q_{\alpha 2}+\tilde{e} . q_{\alpha 1}, \\
\frac{p_{\beta 1}}{m_{\beta}} & \equiv-\nabla_{q_{\beta 1}}\left(\frac{S_{\beta}}{m_{\beta}}\right)=-b-2 d . q_{\beta 1}-e . q_{\beta 2}, \\
\frac{p_{\beta 2}}{m_{\beta}} & \equiv \nabla_{q_{\beta 2}}\left(\frac{S_{\beta}}{m_{\beta}}\right)=c+2 f . q_{\beta 2}+\tilde{e} . q_{\beta 1}
\end{aligned}
$$

and obtain the following expression:

$$
\frac{S_{\alpha}}{m_{\alpha}}-\frac{S_{\beta}}{m_{\beta}}=\frac{1}{2}\left(\frac{p_{\alpha 2}}{m_{\alpha}}+\frac{p_{\beta 2}}{m_{\beta}}\right) \cdot\left(q_{\alpha 2}-q_{\beta 2}\right)-\frac{1}{2}\left(\frac{p_{\alpha 1}}{m_{\alpha}}+\frac{p_{\beta 1}}{m_{\beta}}\right) \cdot\left(q_{\alpha 1}-q_{\beta 1}\right) .
$$

The same relation holds for the classical action concerning two actual paths with a common drift time (homologous paths). This yields an other demonstration of the first theorem expressed in Section 4.

\section{References}

[1] P. Berman (Ed.), Atom Interferometry, Academic Press, San Diego, 1997.

[2] Ch.J. Bordé, C. R. Acad. Sci. Paris Série IV 2 (2001) 509.

[3] Ch.J. Bordé, Metrologia 39 (5) (2002) 435.

[4] Ch.J. Bordé, An elementary quantum theory of atom-wave beam splitters: the ttt theorem, Lecture Notes for a Mini-Course, Institut für Quantenoptik, Universität Hannover, 2002, to be published.

[5] J. Audretsch, K.-P. Marzlin, J. Phys. II (France) 4 (1994) 2073.

[6] Ch. Antoine, Ch.J. Bordé, J. Opt. B, submitted for publication.

[7] A. Peters, K.Y. Chung, S. Chu, Metrologia 38 (2001) 25.

[8] M.J. Snadden, J.M. McGuirk, P. Bouyer, K.G. Haritos, M.A. Kasevich, Phys. Rev. Lett. 81 (1998) 971.

[9] P. Wolf, Ph. Tourrenc, Phys. Lett. A 251 (1999) 241.

[10] R. Bingham, et al., HYPER, Hyper-precision cold atom interferometry in space, Assessment Study report, ESA-SCI (2000).

[11] Ch.J. Bordé, Phys. Lett. A 140 (1989) 59.

[12] Ch.J. Bordé, Atomic interferometry and laser spectroscopy, in: Laser Spectroscopy X, World Scientific, Singapore, 1991 , pp. $239-245$.

[13] J. Ishikawa, F. Riehle, J. Helmcke, Ch.J. Bordé, Phys. Rev. A 49 (1994) 4794. 